\title{
Estimation of the internal shear strength distribution of the element for laminated veneer lumber by nonlinear least-squares method
}

\author{
Makoto Koseki ${ }^{1} \cdot$ Noboru Nakamura ${ }^{2} \cdot$ Shinichiro $\mathrm{Naoi}^{3}$
}

Received: 12 January 2018 / Accepted: 12 April 2018 / Published online: 22 May 2018

(c) The Japan Wood Research Society 2018

\begin{abstract}
Until now we developed an estimation method for strength distributions of laminated veneer lumber (LVL) element by nonlinear least-squares method (NLM). Estimated strengths by this method were modulus of elasticity (MOE) and modulus of rupture (MOR) in the horizontal use direction and the vertical use direction, tensile strength and compression strength. But to use LVL for structural members, shear strength was also needed. Therefore, we tried to estimate the shear strength distribution of LVL element by NLM same as MOE and MOR in the horizontal use direction and the vertical use direction, the tensile strength of LVL and the compression strength of LVL in the previous reports. We conducted shear strength test for LVL and estimated element shear strength distribution by LVL strength data in the horizontal and vertical use direction. Next, we simulated LVL shear strength distribution using element shear strength distribution and compared with experimental ones in each use direction. They were overlapped in both use direction. Therefore, we could validate NLM for estimating element shear strength distribution.
\end{abstract}

Keywords Laminated veneer lumber (LVL) · Monte Carlo simulation · Nonlinear least-squares method · Element of LVL . Shear strength distribution

\section{Introduction}

Many researches [1-3] for estimating the glulam strength have been reported. In these reports, the element of glulam is considered as lamina and adhesive layers are ignored. If we can get the strength distribution of lamina, it is possible to estimate the glulam strength distribution based on some failure criterion, for example, the equation including bending and tensile stress or tensile stress only is supposed.

On the other hand, there was no research for estimating laminated veneer lumber (LVL) strength. Therefore, we

Part of this report was presented at the Annual Meeting of the Architectural Institute of Japan, Fukuoka, Japan, August 2016.

Makoto Koseki

m.koseki@jpic-ew.or.jp

1 Japan Plywood Inspection Corporation, 2-11-33 Yatsuka, Soka-shi, Saitama 340-0028, Japan

2 Institute of Wood Technology, Akita Prefectural University, Noshiro 016-0876, Japan

3 WOOD ONE CO., LTD, 1-12-1 Shimohera, Hatsukaichi 738-0023, Hiroshima, Japan developed the method [4-6] for estimating LVL strength by nonlinear least-squares method (NLM). In this method, we supposed the element as veneer with adhesive. Because adhesive enters into lathe checks and we cannot use veneer strengths before gluing. And we could estimate modulus of elasticity (MOE) and modulus of rupture (MOR) in horizontal and vertical use direction, tensile and compression strength. But to use LVL for structural members, shear strength of LVL is also needed. Therefore, we tried to estimate the shear strength distribution of LVL element by NLM. First, we supposed the criterion of LVL shear strength in horizontal and vertical use direction. Second, we conducted shear strength test for LVL and estimated the element shear strength distributions. Third, we simulated LVL shear strength distribution using element shear strength distribution and compared simulated one with experimental one in each use direction. Finally, we made sure that they were overlapped in both use direction. Therefore, we could validate NLM for estimating the element shear strength distribution.

From the view point of actual use, the necessity of NLM estimation of the element strength distribution is shown as follows. MOE classifications of normally produced LVL are 
$60 \mathrm{E}$ in sugi, $80 \mathrm{E}$ in cypress, $100 \mathrm{E}$ in radiata pine, $120 \mathrm{E}$ in larch and 140E in Dahurian larch [7] in Japan. On the other hand, average MOEs of small clear specimens are $7.5 \mathrm{kN} / \mathrm{mm}^{2}$ in sugi, $9.0 \mathrm{kN} / \mathrm{mm}^{2}$ in cypress, $8.5 \mathrm{kN} / \mathrm{mm}^{2}$ in radiata pine, $10.0 \mathrm{kN} / \mathrm{mm}^{2}$ in larch and $12.0 \mathrm{kN} / \mathrm{mm}^{2}$ in Dahurian larch [8]. Therefore, we can see MOEs of normally produced LVL are higher than average MOEs of small clear specimens except for sugi. In LVL production companies, veneers are classified by the supersonic propagation velocity from which Young's modulus can be calculated by supposing average density. From these facts, it is the present situation that veneers with higher propagation velocity, higher classified veneer in other words, are used to manufacture LVL in JAS (Japanese Agricultural Standard for laminated veneer lumber) [9]. In these cases, lower classified veneer are not necessarily used. In the end, the yield rate is low. For using lower classified veneer, LVL is necessarily composed of different classified veneers. When the element strength distributions can be estimated by NLM, LVL production companies can use lower classified veneer. And one LVL production company is now trying to do so.

\section{Estimation of element shear strength distribution by NLM}

\section{Shear strength in the vertical use direction}

The schematic diagram of shear force in cross section $\mathrm{A}^{-\mathrm{A}^{\prime}}$ caused by the moment $M$ in the vertical use direction for $n$ layers LVL is shown in Fig. 1. $M_{i}(i=1, \ldots, n)$ is supposed as the bending moment in the $i$ th element, veneer with adhesive in other words, composing LVL. The sum of $M_{i}$ makes $M$ loaded to LVL. Suppose the shear force caused to $i$ th element and LVL are $Q_{i}$ and $Q_{\mathrm{V}}$, respectively, we can get the following equation:

$Q_{i}=\frac{E_{i} I_{i}}{(E I)_{\mathrm{V}}} Q_{\mathrm{V}} \quad(i=1, \ldots, n)$

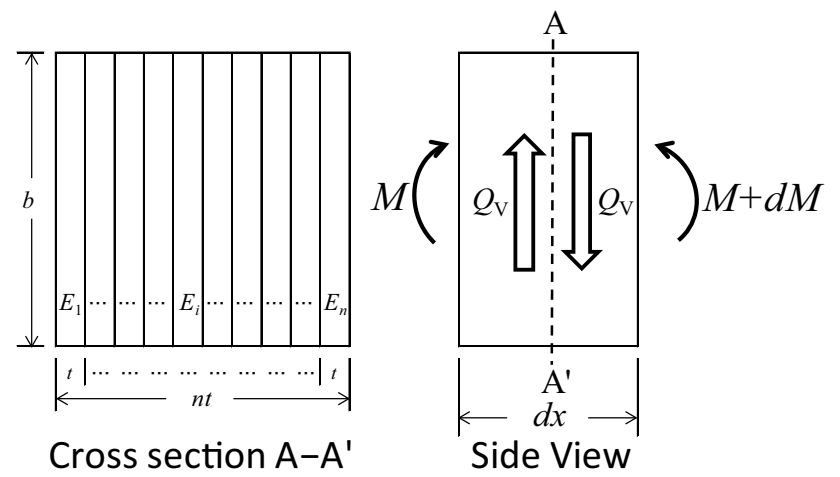

Fig. 1 The schematic diagram of shear force caused by the moment $M$ in the vertical use direction. Notes: $E_{i}: \mathrm{MOE}$ in $i$ th layer where $E_{i}$ is MOE of $i$ th element, $I_{i}$ is the moment of inertia of $i$ th element, $(E I)_{\mathrm{V}}$ is the stiffness of LVL in the vertical use direction. Suppose the width and thickness of $i$ th element are $b$ and $t$, respectively, Eq. (1) is transformed to Eq. (2).

$Q_{i}=\frac{E_{i}}{\sum_{k=1}^{n} E_{k}} Q_{\mathrm{V}} \quad(i=1, \ldots, n)$

The maximum shear stress of $i$ th element and LVL are shown as $\frac{3 Q_{i}}{2 t b}$ and $\frac{3 Q_{\mathrm{V}}}{2 n t b}$, respectively. Suppose $i$ th element and LVL fails at these stresses, we can get the following equation:

$\frac{2 t b \tau_{i}}{3}=\frac{E_{i}}{\sum_{k=1}^{n} E_{k}} \frac{2 n t b \tau_{\mathrm{V}}}{3} \quad(i=1, \ldots, n)$

where $\tau_{i}$ is the shear strength of $i$ th element and $\tau_{\mathrm{V}}$ is the shear strength of LVL. Equation (3) is transformed to the following equation:

$\tau_{\mathrm{V}}=\frac{\sum_{k=1}^{n} E_{k}}{n} \frac{\tau_{i}}{E_{i}} \quad(i=1, \cdots, n)$

Then suppose the failure criterion of LVL is that one element fails first by shear stress, we can show shear strength of LVL by the following equation from Eq. (4).

$\tau_{\mathrm{V}}=\min \left\{E_{\mathrm{V}(\mathrm{LVL})}\left(\frac{\tau_{i}}{E_{i}}\right)\right\} \quad(i=1, \ldots, n)$

where $E_{\mathrm{v}(\mathrm{LVL})}$ is MOE of LVL in the vertical use direction, $\min \left\{{ }^{*}\right\}$ is the smallest value in the braces.
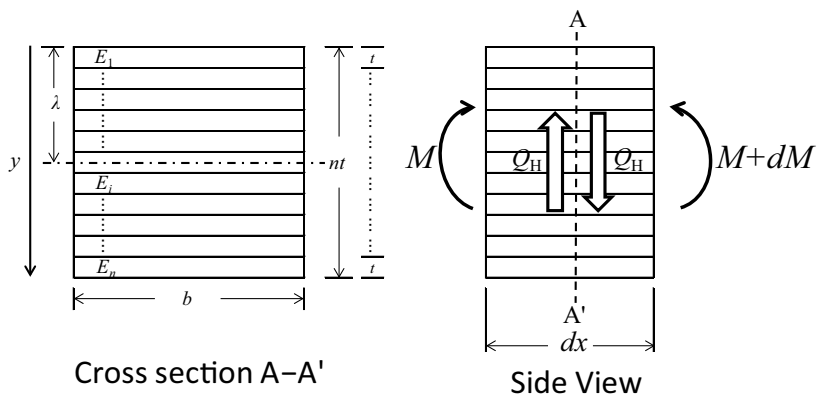

Fig. 2 The schematic diagram of shear force caused by the moment $M$ in the horizontal use direction. Notes: $E_{i}$ : MOE in $i$ th layer, $\lambda$ : the distance of neutral axis from the upper side 


\section{Shear strength in the horizontal use direction}

The schematic diagram of shear force in cross section $\mathrm{A}-\mathrm{A}^{\prime}$ caused by the moment $M$ in the horizontal use direction for $n$ layers LVL is shown in Fig. 2. By the elementary theory of beam, the shear stress $\tau(s)$ of $i$ th element at the arbitrary position $s((i-1) t \leqq s \leqq i t)$ from the upper side of LVL is shown by the following equation:

$\tau(s)_{i}=\frac{Q_{\mathrm{H}}}{(E I)_{\mathrm{H}}}\left\{\int_{s}^{i t} E_{i}(y-\lambda) \mathrm{d} y+\sum_{j=i+1}^{n} \int_{(j-1) t}^{j t} E_{j}(y-\lambda) \mathrm{d} y\right\}$

where $s((i-1) t \leqq s \leqq i t)$ is located in the $i$ th element, $E_{i}$ is MOE of $i$ th element, $(E I)_{\mathrm{H}}$ is stiffness of LVL in the horizontal use direction and $\lambda$ is the distance of neutral axis of LVL from the upper side of LVL. Suppose the average shear stress of $i$ th element is $\bar{\tau}_{i}$, we can get the following equation:

$$
\begin{aligned}
\bar{\tau}_{i} & =\frac{1}{i t-(i-1) t} \int_{(i-1) t}^{i t} \tau(s)_{i} \mathrm{~d} s \\
& =\frac{Q_{\mathrm{H}}}{t(E I)_{\mathrm{H}}}\left\{E_{i} \int_{(i-1) t}^{i t} \int_{s}^{i t}(y-\lambda) \mathrm{d} y \mathrm{~d} s+t \sum_{j=i+1}^{n} E_{j} \int_{(j-1) t}^{i t}(y-\lambda) \mathrm{d} y\right\}
\end{aligned}
$$

Then the first integral term in the right side of Eq. (6) is calculated into the following equation:

$$
\begin{aligned}
\int_{(i-1) t}^{i t} \int_{s}^{i t}(y-\lambda) \mathrm{d} y \mathrm{~d} s & =\int_{(i-1) t}^{i t}\left(-\frac{s^{2}}{2}+\lambda s+\frac{i^{2} t^{2}}{2}-\lambda i t\right) \mathrm{d} s \\
= & \frac{i t^{3}}{2}-\frac{t^{3}}{6}-\frac{\lambda t^{2}}{2}
\end{aligned}
$$

And then the second integral term in the right side of Eq. (6) is calculated into the following equation:

$$
\int_{(j-1) t}^{i t}(y-\lambda) \mathrm{d} y=\left(j t-\lambda-\frac{t}{2}\right) t
$$

Finally, Eq. (6) is terminated to the following equation from Eqs. (7) and (8).

$$
\bar{\tau}_{i}=\frac{Q_{\mathrm{H}}}{(E I)_{\mathrm{H}}}\left\{E_{i}\left(\frac{i t}{2}-\frac{t}{6}-\frac{\lambda}{2}\right)+\sum_{j=i+1}^{n} E_{j}\left(j t-\lambda-\frac{t}{2}\right)\right\}
$$

Then suppose the shear strength of LVL is $\tau_{\mathrm{H}}$ and $\tau_{\mathrm{H}}$ is calculated by the equation $\tau_{\mathrm{H}}=\frac{3 Q_{\mathrm{H}}}{2 n t b}$ when the $i$ th element is failed by the shear stress $\bar{\tau}_{i}$, we can get the following equation:

$$
\tau_{\mathrm{H}}=\frac{3 \bar{\tau}_{i}}{2 n t^{2} b} \frac{(E I)_{\mathrm{H}}}{E_{i}\left(\frac{i t}{2}-\frac{t}{6}-\frac{\lambda}{2}\right)+\sum_{j=i+1}^{n} E_{j}\left(j t-\lambda-\frac{t}{2}\right)}
$$

Now suppose the failure criterion of LVL is that one element fails first by shear stress, we can get the following equation from Eq. (10).

$$
\begin{aligned}
& \tau_{\mathrm{H}}= \min \left\{\frac{3 \bar{\tau}_{i}}{2 n t^{2} b} \frac{(E I)_{\mathrm{H}}}{E_{i}\left(\frac{i t}{2}-\frac{t}{6}-\frac{\lambda}{2}\right)+\sum_{j=i+1}^{n} E_{j}\left(j t-\lambda-\frac{t}{2}\right)}\right\} \\
&(i=1, \ldots, n)
\end{aligned}
$$

\section{Algorithm to estimate parameters for element strength distribution}

\section{Element MOE distribution in the vertical use direction}

For example, if the element MOE distribution is supposed as normal distribution, parameters are shown by the twodimensional vector $\left(E_{\mathrm{Vavg}}, E_{\mathrm{Vstd}}\right) . E_{\mathrm{Vavg}}$ and $E_{\mathrm{Vstd}}$ are mean value and standard deviation of element MOE in the vertical use direction. The algorithm to calculate this vector is shown as follows.

(1) The initial values $\left(P_{1}, P_{2}\right)$ are given to $\left(E_{\mathrm{Vavg}}, E_{\mathrm{Vstd}}\right)$.

(2) The number of layers for LVL is set as $n$ and the number of test sample is also set as $N$. MOE of $r$ th test sample is $E_{\mathrm{e}, r}$ and the simulated MOE is $E_{\mathrm{c}, r}$ corresponding to $E_{\mathrm{e}, r}$.

(3) Normal random variable $E_{r, i}$ according to $\left(E_{\mathrm{Vavg}}, E_{\mathrm{Vstd}}\right)$ of $i$ th element in $r$ th test sample is produced by generating uniform random variables $U_{r, i}\left(0 \leqq U_{r, I}<1\right)$.

(4) Repeat (3) $n$ times and calculate MOE of LVL, $E_{\mathrm{Vc}, r}$, by the following equation:

$E_{\mathrm{Vc}, r}=\frac{\sum_{i=1}^{n} E_{r, i}}{n}$

From now on, $U_{r, i}$ are fixed.

(5) Make $S_{\mathrm{e}}$, the residual square sum expressed by the following equation, minimum by changing $P_{1}$ and $P_{2}$. The convergent value is $\left(E_{\mathrm{Vavg}}, E_{\mathrm{Vstd}}\right)$. 


$$
S_{\mathrm{e}}=\sum_{r=1}^{N}\left(E_{\mathrm{e}, r}-E_{\mathrm{c}, r}\right)^{2}
$$

(6) Confirm the convergence of $\left(E_{\mathrm{Vavg}}, E_{\mathrm{Vstd}}\right)$ as the solution of the normal equation by the following partial differentiation coefficients.

$$
\frac{\partial S_{\mathrm{e}}}{\partial E_{\mathrm{Vavg}}} \cong 0, \frac{\partial S_{\mathrm{e}}}{\partial E_{\mathrm{Vstd}}} \cong 0
$$

\section{Element shear strength distribution in the vertical use direction}

For example, if the element shear strength distribution, $F_{\mathrm{V}}$, is supposed as normal distribution, parameters are shown by the three-dimensional vector $\left(F_{\mathrm{Vavg}}, F_{\mathrm{Vstd}}, R_{\mathrm{EV}-\mathrm{FV}}\right)$. $F_{\text {Vavg }}$ and $F_{\text {Vstd }}$ are mean value and standard deviation of element shear strength in the vertical use direction. $R_{\mathrm{EV}-\mathrm{FV}}$ is the correlation coefficient between MOE and shear strength. Therefore, the algorithm to calculate ( $F_{\text {Vavg, }}$, $\left.F_{\mathrm{Vstd}}, R_{\mathrm{EV}-\mathrm{FV}}\right)$ is shown as follows.

(1) The initial values $\left(P_{1}, P_{2}, P_{3}\right)$ are given to $\left(F_{\text {Vavg }}, F_{\text {Vstd }}\right.$, $\left.R_{\mathrm{EV}-\mathrm{FV}}\right)$.

(2) The number of layers for LVL is set as $n$ and the number of test sample is set as $N$. Shear strength of $r$ th test sample is $F_{\mathrm{Ve}, r}$ and the simulated shear strength is $F_{\mathrm{Vc}, r}$ corresponding to $F_{\mathrm{Ve}, r}$.

(3) Generate uniform random variables $U_{\mathrm{e}, r, i}\left(0 \leqq U_{\mathrm{e}, r, i}<1\right)$ for $E_{r, i}$ and $U_{\mathrm{f}, r, i}\left(0 \leqq U_{\mathrm{f}, r, i}<1\right)$ for $F_{\mathrm{V} .} U_{\mathrm{e}, r, i}$ and $U_{\mathrm{f}, r, i}$ are mutually independent.

(4) Normal random variable $E_{r, i}$ according to $\left(E_{\mathrm{Vavg}}, E_{\mathrm{Vstd}}\right)$ is produced using $U_{\mathrm{e}, r, i}$ shown in the previous section. And then correlated normal random variable $F_{\mathrm{s}, i}$ with $E_{\mathrm{s}, i}$ according to $\left(F_{\mathrm{Vavg}}, F_{\mathrm{Vstd}}, R_{\mathrm{EV}-\mathrm{FV}}\right)$ is produced using $U_{\mathrm{e}, r, i}, U_{\mathrm{f}, r, i}$ and $\left(P_{1}, P_{2}, P_{3}\right)$.

(5) Repeat from (3) to (4) $n$ times and calculate shear strength, $F_{\mathrm{Vc}, r}$ by the following equation:

$F_{\mathrm{Vc}, r}=\min \left\{\frac{F_{\mathrm{s}, i}}{E_{\mathrm{s}, i}} E_{\mathrm{Vc}, r}\right\}$

From now on, $U_{\mathrm{e}, r i}$ and $U_{\mathrm{f}, r, i}$ are fixed.

(6) Make $S_{\mathrm{f}}$, the residual square sum expressed by the following equation, minimum by changing $P_{1}, P_{2}$ and $P_{3}$. The convergent value is ( $\left.F_{\text {Vavg }}, F_{\mathrm{Vstd}}, R_{\mathrm{EV}-\mathrm{FV}}\right)$.

$S_{\mathrm{f}}=\sum_{r=1}^{N}\left(F_{\mathrm{Ve}, r}-F_{\mathrm{Vc}, r}\right)^{2}$
(7) Confirm the convergence of $\left(F_{\mathrm{Vavg}}, F_{\mathrm{Vstd}}, R_{\mathrm{EV}-\mathrm{FV}}\right)$ as the solution of the normal equation by the following partial differentiation coefficients.

$$
\frac{\partial S_{\mathrm{f}}}{\partial F_{\mathrm{Vavg}}} \cong 0, \frac{\partial S_{\mathrm{f}}}{\partial F_{\mathrm{Vstd}}} \cong 0, \frac{\partial S_{\mathrm{f}}}{\partial R_{\mathrm{EV}-\mathrm{FV}}} \cong 0
$$

\section{Element MOE distribution in the horizontal use direction}

For example, if the element MOE distribution is supposed as normal distribution, parameters are shown by the two-dimensional vector $\left(E_{\mathrm{Havg}}, E_{\mathrm{Hstd}}\right) . E_{\mathrm{Havg}}$ and $E_{\mathrm{Hstd}}$ are mean value and standard deviation of element MOE in the horizontal use direction. The algorithm to calculate this vector is same as that for calculating element MOE distribution in vertical use direction, however, $E_{\mathrm{Vavg}}$ and $E_{\mathrm{Vstd}}$ are changed to $E_{\mathrm{Havg}}$ and $E_{\mathrm{Hstd}}$, respectively, and the Eq. (12) is changed by the following equation:

$E_{\mathrm{Hc}, r}=\frac{(E I)_{\mathrm{e}, r}}{I}$

where

$$
\begin{array}{r}
(E I)_{\mathrm{e}, r}=\sum_{i=1}^{n} E_{\mathrm{s}, i} I_{i}, I_{i}=\frac{b t^{3}}{12}+g_{i}{ }^{2} b t, g_{i}=\lambda+\left(i-\frac{1}{2}\right) t, \\
\lambda=\frac{1}{2}\left\{\frac{\sum_{i=1}^{n} E_{r, i}(2 i-1)}{\sum_{i=1}^{n} E_{r, i}}\right\}, I=\frac{n^{3} b t^{3}}{12}
\end{array}
$$

where $\lambda$ is the distance of neutral axis of LVL from the upper side of LVL, $g_{i}$ is the distance of $i$ th layer neutral axis from $\lambda, b$ is width of element. $t$ is thickness of element, $I$ is the moment of inertia of LVL, $I_{i}$ is the moment of inertia of $i$ th layer and $(E I)_{\mathrm{e}, r}$ is the bending stiffness of LVL.

\section{Element shear strength distribution in the horizontal use direction}

For example, if the element shear strength distribution is supposed as normal distribution, parameters are shown by the three-dimensional vector $\left(F_{\mathrm{Havg}}, F_{\mathrm{Hstd}}, R_{\mathrm{EV}-\mathrm{FV}}\right) . F_{\mathrm{Havg}}$ and $F_{\mathrm{Hstd}}$ are mean value and standard deviation of element shear strength in the horizontal use direction. $R_{\mathrm{EV}-\mathrm{FV}}$ is the correlation coefficient between MOE and shear strength. The algorithm to calculate parameters in the three-dimensional vector is same as that for calculating element shear strength distribution in vertical use direction, however, $E_{\mathrm{Vavg}}$ and $E_{\mathrm{V} s t d}$ are changed to $E_{\mathrm{Havg}}$ and $E_{\mathrm{Hstd}}$, respectively and Eq. (13) is changed by the following equation: 


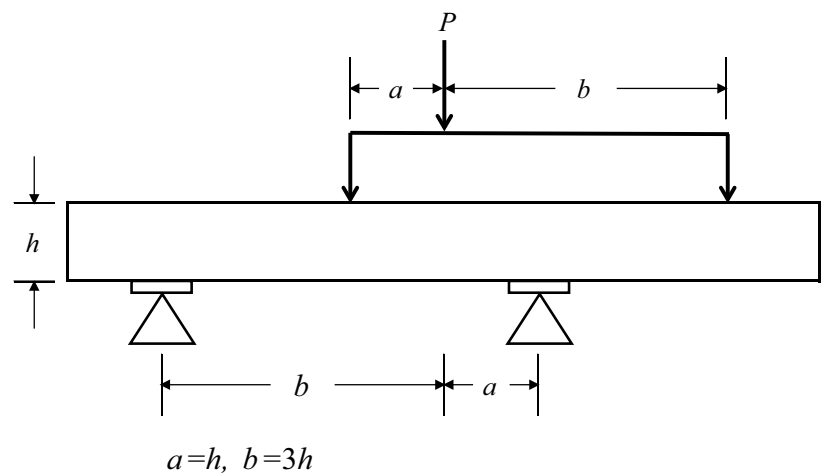

Fig. 3 Antisymmetric 4 point bending for shear test

$$
\begin{gathered}
F_{\mathrm{Hc}, r}=\min \left[\frac{3 \bar{\tau}_{i}}{2 n t^{2} b} \frac{(E I)_{\mathrm{e}, r}}{E_{r, i}\left(\frac{i t}{2}-\frac{t}{6}-\frac{\lambda}{2}\right)+\sum_{j=i+1}^{n} E_{r, j}\left(j t-\lambda-\frac{t}{2}\right)}\right] \\
(i=1, \ldots, n)
\end{gathered}
$$

\section{Material and experiment}

We adopted the antisymmetric 4 points bending test as shown in Fig. 3. The reason is that specimens are more likely broken in shear mode than by the centralized loading test in JAS [9].

We had 47 groups of test specimen. They include 3 species, 8 MOE classifications of LVL JAS [9], a lot of cross sections and various veneer compositions. $8 \mathrm{MOE}$ classifications were $60 \mathrm{E}, 70 \mathrm{E}, 80 \mathrm{E}, 90 \mathrm{E}, 110 \mathrm{E}, 120 \mathrm{E}, 140 \mathrm{E}$ and $160 \mathrm{E}$. Some veneers have scarf joint and others do not have scarf joint. Composition of veneer, shear test use direction and cross section of LVL specimen for each test number are shown in Table 1. Species and JAS classification of LVL of all test number are radiata pine (Pinus radiata) and 110E [9], respectively. All veneers of LVL did not include scarf joint.

In many groups, main failure mode was bending. In such case, we cannot precisely estimate element shear strength distribution. Therefore, we used only groups where failure mode was shear.
Table 2 Results of laminated veneer lumber shear test

\begin{tabular}{llrllll}
\hline Test no. & $\begin{array}{l}\text { Number of } \\
\text { specimens }\end{array}$ & \multicolumn{2}{l}{$\begin{array}{l}\text { Modulus of elasticity } \\
\left(\mathrm{kN} / \mathrm{mm}^{2}\right)\end{array}$} & & \multicolumn{2}{l}{$\begin{array}{l}\text { Shear strength } \\
\left(\mathrm{N} / \mathrm{mm}^{2}\right)\end{array}$} \\
\cline { 6 - 7 } & & Average & $\begin{array}{l}\text { Standard } \\
\text { deviation }\end{array}$ & Average & $\begin{array}{l}\text { Standard } \\
\text { deviation }\end{array}$ \\
\hline 1 & 21 & 12.03 & 1.36 & 7.37 & 0.92 \\
2 & 12 & 9.54 & 1.23 & 6.32 & 1.07 \\
3 & 18 & 11.73 & 1.05 & 5.67 & 0.70 \\
4 & 21 & 11.44 & 0.77 & 6.09 & 0.71 \\
5 & 18 & 11.18 & 0.68 & 5.99 & 0.45 \\
\hline
\end{tabular}

\section{Result and discussion}

The results of shear test are shown in Table 2. From the data, we estimated element MOE distribution and element shear strength distribution by NLM. For each distribution, we supposed normal distribution, log-normal distribution and 2-P Weibull distribution [10]. Using these distribution, we calculated the residual of sum of squares. Finally, we chose the distribution whose residual sum of squares was the smallest. We considered the parameters as the solution of the normal equation, because the partial differentiation coefficients above mentioned were almost zero. Parameters of them are shown in Table 3. Next, we validated these parameters as follows.

(1) Using estimated parameters we generated $n$ element MOEs and shear strengths for $n$ layers LVL.

(2) We calculated MOE and shear strength by Eqs. (5) and (11).

(3) Repeating from (1) to (2) 500 times, we generated 500 MOEs and shear strengths for $n$ layers LVL.

(4) We sorted 500 MOEs and shear strengths in ascending order and calculated the cumulative probability by median rank method. And for the experimental data, we also sorted in ascending order and calculated the cumulative probability by median rank method.

(5) Finally, we compared them.

The cumulative probability of shear strength in No. 3 is shown as an example in Fig. 4. They are almost same. We
Table 1 Outline of laminated veneer lumber test sample

\begin{tabular}{llll}
\hline Test no. & Direction of use & Composition of veneer & Cross section of the specimen \\
\hline 1 & Horizontal & $4.3 \mathrm{~mm} \times 5 \mathrm{ply}$ & $\mathrm{T} 21 \mathrm{~mm} \times \mathrm{W} 90 \mathrm{~mm}$ \\
2 & Ditto & $4.3 \mathrm{~mm} \times 8 \mathrm{ply}$ & $\mathrm{T} 33 \mathrm{~mm} \times \mathrm{W} 90 \mathrm{~mm}$ \\
3 & Ditto & $4.3 \mathrm{~mm} \times 11 \mathrm{ply}$ & $\mathrm{T} 46 \mathrm{~mm} \times \mathrm{W} 90 \mathrm{~mm}$ \\
4 & Vertical & $4.3 \mathrm{~mm} \times 8 \mathrm{ply}$ & $\mathrm{T} 33 \mathrm{~mm} \times \mathrm{W} 33 \mathrm{~mm}$ \\
5 & Ditto & $4.3 \mathrm{~mm} \times 11 \mathrm{ply}$ & $\mathrm{T} 46 \mathrm{~mm} \times \mathrm{W} 46 \mathrm{~mm}$ \\
\hline
\end{tabular}

$T$ thickness, $W$ width 
Table 3 The values of parameter for the element estimated strengths by nonlinear least-squares method

\begin{tabular}{|c|c|c|c|c|c|c|c|c|}
\hline \multirow[t]{2}{*}{ Test no. } & \multicolumn{2}{|l|}{ Distribution } & \multicolumn{2}{|c|}{ Modulus of elasticity } & \multicolumn{2}{|l|}{ Shear strength } & \multirow{2}{*}{$\begin{array}{l}\text { Correlation } \\
\text { coefficient }\end{array}$} & \multirow{2}{*}{$\begin{array}{l}\text { Residual } \\
\text { sum of } \\
\text { squares }\end{array}$} \\
\hline & $\begin{array}{l}\text { Modulus of } \\
\text { elasticity }\end{array}$ & Shear strength & Parameter 1 & Parameter 2 & Parameter 1 & Parameter 2 & & \\
\hline 1 & $2 \mathrm{PW}$ & $\mathrm{LN}$ & $13.05\left(\mathrm{kN} / \mathrm{mm}^{2}\right)$ & 6.04 & 2.08 & 0.20 & -0.98 & 0.07 \\
\hline 2 & $\mathrm{LN}$ & $\mathrm{N}$ & 2.25 & 0.22 & $7.61\left(\mathrm{~N} / \mathrm{mm}^{2}\right)$ & $1.68\left(\mathrm{~N} / \mathrm{mm}^{2}\right)$ & -0.95 & 0.04 \\
\hline 3 & $\mathrm{LN}$ & $2 \mathrm{PW}$ & 2.44 & 0.22 & $6.42\left(\mathrm{~N} / \mathrm{mm}^{2}\right)$ & 11.91 & 0.77 & 0.04 \\
\hline 4 & $2 \mathrm{PW}$ & $\mathrm{N}$ & $12.16\left(\mathrm{kN} / \mathrm{mm}^{2}\right)$ & 6.39 & $8.20\left(\mathrm{~N} / \mathrm{mm}^{2}\right)$ & $0.98\left(\mathrm{~N} / \mathrm{mm}^{2}\right)$ & -0.01 & 0.08 \\
\hline 5 & LN & LN & 2.39 & 0.21 & 2.04 & 0.07 & 0.64 & 0.03 \\
\hline
\end{tabular}

$N$ normal distribution, $L N$ log-normal distribution, $2 P W$ 2-parameter Weibull distribution, Parameter 1 average in case of $\mathrm{N}$ and $\mathrm{LN}$, scale parameter in case of $2 \mathrm{PW}$, Parameter 2 standard deviation in case of $\mathrm{N}$ and $\mathrm{LN}$, shape parameter in case of $2 \mathrm{PW}$

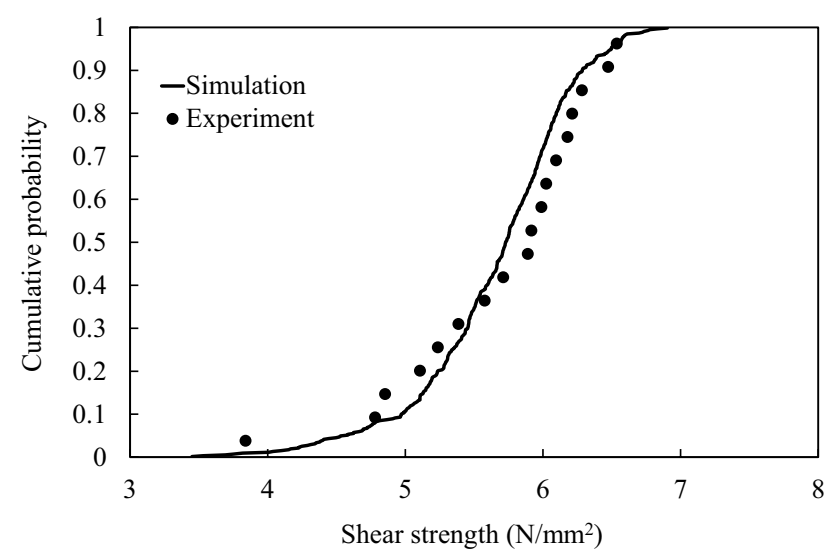

Fig. 4 Cumulative probability of simulated and experimental strength in case No. 3

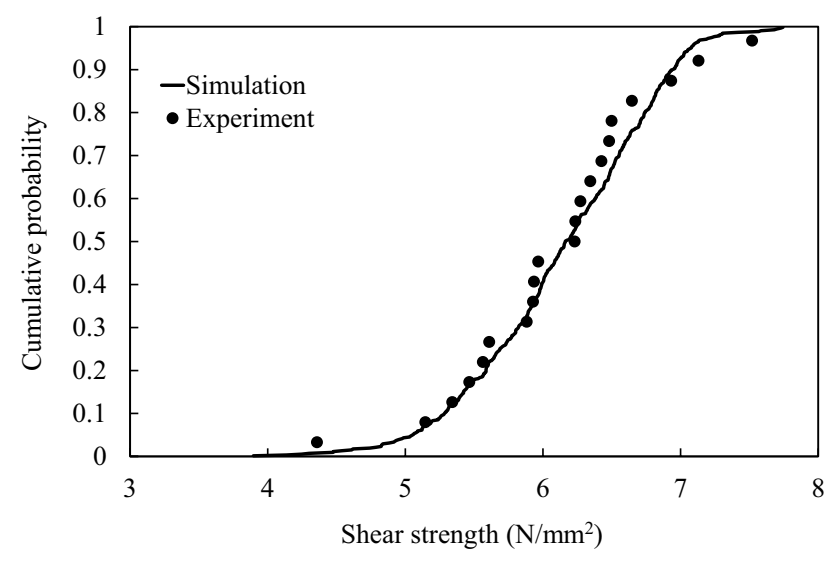

Fig. 5 Cumulative probability of simulated and experimental strength in case No. 4

confirm they are also almost same in case of Nos. 1 and 2. The cumulative probability of shear strength in No. 4 is shown as an example in Fig. 5. We can see they are almost same. We confirm they are also almost same in case No. 5. Next, we show relationships between MOE and shear

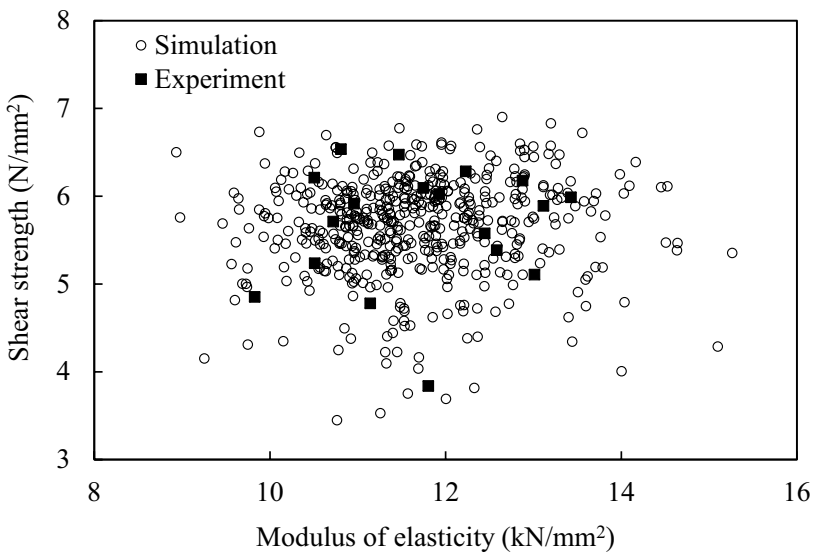

Fig. 6 Relationship between modulus of elasticity and shear strength in case No. 3

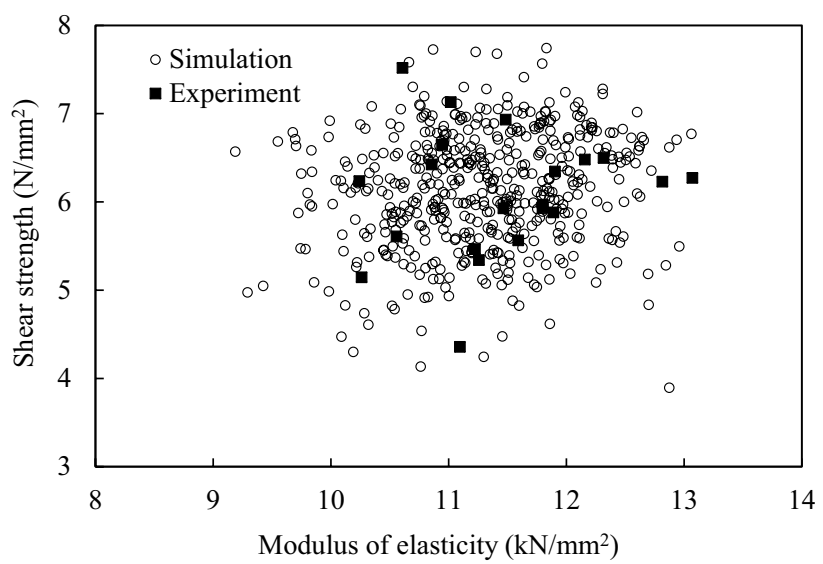

Fig. 7 Relationship between modulus of elasticity and shear strength in case No. 4

strength in case Nos. 3 and 4 in Figs. 6 and 7, respectively. Simulated data overlap experiment data. We confirm they also overlap in case Nos. 1, 2 and 5 . 
In the previous reports $[5,6]$, we extracted specimens of 1 ply, 2 ply and 3 ply with an adhesion layer from the remainders of LVL and carried out the same static tests as the experiments for LVL. But it is almost impossible to conduct the shear test of 1 ply specimen in horizontal and vertical use direction. If it is possible to conduct the shear test of 1 ply specimen and we find laminating effect exists, we can do the same analysis by comparing 1 ply shear strength distribution including laminating effect with element shear strength distribution as shown in previous reports [5, 6]. Consequently, we can think NLM is valid for estimating element shear strength distribution.

\section{Conclusion}

We conducted the shear strength test for LVL and estimated the shear strength distribution of LVL element by NLM same as MOR in the horizontal and vertical use direction, tensile strength and compression strength published in the previous reports [4-6]. Next, we simulated the shear strength distribution of LVL using the estimated shear strength distribution of LVL element and compared the simulated strength distribution with experimental strength distribution in the horizontal and vertical use direction. As a result, they overlapped in each use direction. Therefore, we could validate NLM for estimating element shear strength distribution.

\section{References}

1. Foschi RO, Barrett JD (1980) Glued-laminated beam strength: a model. J Struct Div 106:1735-1754

2. Hernandez R, Bender DA, Richburg BA, Kline KS (1992) Probabilistic modeling of glued-laminated timber beams. Wood Fiber Sci 24:294-306

3. Komatsu K (1997) Prediction of maximum bending moment of glulam beam composed of arbitrary laminae and verification by experiment results (in Japanese). Mokuzai Gakkaishi 43:934-939

4. Koseki M, Nakamura N (2012) Estimation of the strength distribution of elements for laminated veneer lumber by a nonlinear leastsquares method (in Japanese). Mokuzai Gakkaishi 58:125-136

5. Koseki M, Nakamura N (2015) Validation of method for estimating element strength distribution of laminated veneer lumber I (in Japanese). Mokuzai Gakkaishi 61:55-66

6. Koseki M, Nakamura N (2015) Validation of the estimation method of the element strength distribution of laminated veneer lumber II (in Japanese). Mokuzai Gakkaishi 61:371-378

7. Narita T, Lee W, Paku T (2017) The feature and the application example of LVL. In: Okano K (ed) Wood wood based materials and timber construction technologies (in Japanese). NTS Co. Ltd., Tokyo, p 75

8. Forestry and Forest Products Research Institute (ed) (2004) Handbook of wood industry (in Japanese). Maruzen, Tokyo, pp 194-199

9. Japan Agriculture Standard (2013) JAS for laminated veneer lumber (in Japanese), Japan Plywood Inspection Co., Tokyo

10. Horie K (1997) Probability and statistical method of wood strength data (In Japanese). Timber Engineering Institute, Sunagawa, pp 1.5-1.9 